\title{
Design of two-dimensional FIR digital filters with specified magnitude and group delay responses by analytical least-squares method
}

\author{
Soo-Chang Pei ${ }^{\mathrm{a}, *}$, Jong-Jy Shyu ${ }^{\mathrm{b}}$ \\ ${ }^{a}$ Department of Electrical Engineering, National Taiwan University, Taipei, Taiwan 106, ROC \\ ${ }^{b}$ Department of Computer Science and Engineering, Tatung Institute of Technology, Taipei, Taiwan, ROC
}

Received 7 September 1995; revised 21 March 1996 and 4 July 1996

\begin{abstract}
In this paper, an analytical least-squares approach to the design of quadrantally symmetric 2-D filters is extended to design complex-coefficient 2-D FIR filters with arbitrary complex frequency responses. Given the design specifications, the filter coefficients are determined directly from a closed-form transformation matrix, such that time-consuming optimization, matrix inversion and iteration procedures can be avoided. Several examples are presented to demonstrate the simplicity and effectiveness of the proposed method.
\end{abstract}

\section{Zusammenfassung}

In dieser Arbeit wird eine analytische Methode für den Entwurf von quadranten-symmetrischen 2-D Filtern nach dem Kriterium der kleinsten Quadrate auf den Entwurf von 2-D FIR-Filtern mit komplexwertigen Koeffizienten und mit beliebigem komplexem Frequenzgang erweitert. Bei gegebenen Entwurfsspezifikationen werden die Filterkoeffizienten direkt aus einer in geschlossener Form gegebenen Transformationsmatrix bestimmt, so daß zeitaufwendige Optimierung, Matrizeninversion und Iterationsprozesse vermieden werden können. Es werden einige Beispiele vorgestellt, die die Einfachheit und Effizienz der vorgeschlagenen Methode veranschaulichen.

\section{Résumé}

Dans cet article, on généralise une approche analytique de conception de filtres symétriques 2-D par les moindres carrés, afin de créer des filtres RIF 2-D à coefficients complexes et à réponse en fréquence complexe et arbitraire. Etant données les spécifications de conception, les coefficients du filtre sont déterminés directement à partir d'une matrice de transformation fermée, ce qui fait que des procédures prenant du temps telles que l'optimisation, l'inversion de matrices, l'itération peuvent être évitées. Plusieurs exemples sont présentés pour démontrer la simplicité et l'efficacité de la méthode proposée.

Keywords: Analytical least-squares method; FIR filter; 2-D all-pass equalizer

${ }^{*}$ Corresponding author. Tel.: +886-2-3635251 Ext 321; fax: +886-2-3638247; e-mail: pei@cc.ee.ntu.edu.tw. 


\section{Introduction}

Recently, the design of FIR filters with prescribed magnitude and phase responses is widely studied [2,3,5-7]. For the real coefficient FIR filter design, Chen and Parks converted the complex Chebyshev approximation problem into a real approximation solution, then it was in turn solved by standard linear programming techniques [3]. While the above method needs long computation time, Preuss solved the problem by formulating the approximation problem as an equalization problem [7]. Also Alkhainy et al. proposed an effective duality theory to design FIR filter that best approximates an arbitrary complexvalued frequency response [2]. As to the complex filter design, the eigenfilter approach is used to design complex-coefficient FIR filters with arbitrary complex frequency responses based on the computation of an eigenvector of an appropriate complex Hermitian symmetric and positive-definite matrix $[5,6]$.

In this paper, the proposed algorithm can not only design real-coefficient but also complex-coefficient 2-D FIR filters if both magnitude and phase responses are given simultaneously. In principle, the method is extended from the technique for designing realcoefficient zero-phase 2-D FIR filters by analytical least-squares method [1]. The main trait is that a closed-form transformation matrix is obtained such that the filter's coefficients are determined directly from the given frequency response specifications, which not only simplifies design procedures but also saves much design time. Section 2 presents the problem formulation for designing 2-D FIR complex filters by analytical least-squares method. Design examples including multiband filters and all-pass filters are given in Section 3 to demonstrate the effectiveness of the method. Finally, the conclusions are given in Section 4.

\section{Problem formulation for designing 2-D FIR complex filters by analytical least-squares method}

The frequency response of a 2-D FIR complex filter with length $N_{1} \times N_{2}$ is given by

$F\left(\omega_{1}, \omega_{2}\right)=\sum_{n_{1}=0}^{N_{1}-1} \sum_{n_{2}=0}^{N_{2}-1} a\left(n_{1}, n_{2}\right) \mathrm{e}^{-\mathrm{j} n_{1} \omega_{1}} \mathrm{e}^{-\mathrm{j} n_{2} \omega_{2}}$, where $a\left(n_{1}, n_{2}\right)$ are complex filter coefficients and $a\left(n_{1}, n_{2}\right)=a_{\mathrm{R}}\left(n_{1}, n_{2}\right)+\mathrm{j} a_{\mathrm{I}}\left(n_{1}, n_{2}\right)$ in which $a_{\mathrm{R}}\left(n_{1}, n_{2}\right)$ and $a_{\mathrm{I}}\left(n_{1}, n_{2}\right)$ arc the real and imaginary parts of $a\left(n_{1}, n_{2}\right)$, respectively.

In this paper, the object error function is defined by

$$
\begin{aligned}
E= & \sum_{k_{1}=-M}^{M} \sum_{K_{2}=-M}^{M} \mid F_{\mathrm{d}}\left(\frac{k_{1} \pi}{M}, \frac{k_{2} \pi}{M}\right) \\
& -\left.F\left(\frac{k_{1} \pi}{M}, \frac{k_{2} \pi}{M}\right)\right|^{2} \\
= & \sum_{k_{1}=-M}^{M} \sum_{K_{2}=-M}^{M}\left[F_{\mathrm{d}}\left(\frac{k_{1} \pi}{M}, \frac{k_{2} \pi}{M}\right)\right. \\
& \times\left[F_{\mathrm{d}}\left(\frac{k_{1} \pi}{M}, \frac{k_{2} \pi}{M}\right)-F\left(\frac{k_{1} \pi}{M}, \frac{k_{2} \pi}{M}\right)\right]^{*}
\end{aligned}
$$

where $F_{\mathrm{d}}\left(\omega_{1}, \omega_{2}\right)$ is the desired frequency response and $*$ denotes the complex conjugate operator. It is noted that if a constant group delay is desired, the specification of the phase response will be specified in $F_{\mathrm{d}}\left(\omega_{1}, \omega_{2}\right)$. By matrix notation,

$$
\begin{aligned}
E & =\operatorname{tr}\left[\left(\boldsymbol{F}_{\mathrm{d}}-\boldsymbol{F}\right)^{\mathrm{H}}\left(\boldsymbol{F}_{\mathrm{d}}-\boldsymbol{F}\right)\right] \\
& =\operatorname{tr}\left[\boldsymbol{F}_{\mathrm{d}}^{\mathrm{H}} \boldsymbol{F}_{\mathrm{d}}-\boldsymbol{F}_{\mathrm{d}}^{\mathrm{H}} \boldsymbol{F}-\boldsymbol{F}^{\mathrm{H}} \boldsymbol{F}_{\mathrm{d}}+\boldsymbol{F}^{\mathrm{H}} \boldsymbol{F}\right],
\end{aligned}
$$

where $\mathrm{H}$ denotes the Hermitian transpose operation and

$F_{\mathrm{d}}=\left[F_{\mathrm{d}}\left(\frac{i \pi}{M}, \frac{l \pi}{M}\right),-M \leqslant i, l \leqslant M\right]$

and

$F=\left[F\left(\frac{i \pi}{M}, \frac{l \pi}{M}\right),-M \leqslant i, l \leqslant M\right]$.

Further $\boldsymbol{F}$ can be decomposed as

$\boldsymbol{F}=\boldsymbol{P} \boldsymbol{A} \boldsymbol{Q}^{\mathrm{T}}$,

where $\mathrm{T}$ denotes transpose operation and

$$
\begin{aligned}
& \boldsymbol{A}=\left[a(i, l), \quad 0 \leqslant i \leqslant N_{1}-1, \quad 0 \leqslant l \leqslant N_{2}-1\right], \\
& \boldsymbol{P}=\left[\mathrm{e}^{-\mathrm{j} i l \pi / M}, \quad-M \leqslant i \leqslant M, \quad 0 \leqslant l \leqslant N_{1}-1\right]
\end{aligned}
$$

and

$$
\boldsymbol{Q}=\left[\mathrm{e}^{-\mathrm{j} i l \pi / M},-M \leqslant i \leqslant M, \quad 0 \leqslant l \leqslant N_{2}-1\right] .
$$


For simplification, let $N_{1}=N_{2}=N$, then $\boldsymbol{P}=\boldsymbol{Q}$ and $\boldsymbol{F}=\boldsymbol{P} \boldsymbol{A} \boldsymbol{P}^{\mathrm{T}}$.

We call the matrix $\boldsymbol{P}$ as the frequency response transformation matrix [1].

From (3) and (10),

$$
\begin{aligned}
E=\operatorname{tr}[ & \boldsymbol{F}_{\mathrm{d}}^{\mathrm{H}} \boldsymbol{F}_{\mathrm{d}}-\boldsymbol{F}_{\mathrm{d}}^{\mathrm{H}} \boldsymbol{P} \boldsymbol{A} \boldsymbol{P}^{\mathrm{T}}-\left(\boldsymbol{P} \boldsymbol{A} \boldsymbol{P}^{\mathrm{T}}\right)^{\mathrm{H}} \boldsymbol{F}_{\mathrm{d}} \\
& \left.+\left(\boldsymbol{P} \boldsymbol{A} \boldsymbol{P}^{\mathrm{T}}\right)^{\mathrm{H}} \boldsymbol{P} \boldsymbol{A} \boldsymbol{P}^{\mathrm{T}}\right] .
\end{aligned}
$$

By setting $\partial E / \partial A=0$, minimum error is obtained and the coefficient matrix is given by

$\boldsymbol{A}=\left(\boldsymbol{P}^{\mathrm{H}} \boldsymbol{P}\right)^{-1} \boldsymbol{P}^{\mathrm{H}} \boldsymbol{F}_{\mathrm{d}} \boldsymbol{P}^{*}\left(\boldsymbol{P}^{\mathrm{T}} \boldsymbol{P}^{*}\right)^{-1}$.

Let $\boldsymbol{R}=\boldsymbol{P}^{\mathrm{H}} \boldsymbol{P}$, then

$\boldsymbol{A}=\boldsymbol{R}^{-1} \boldsymbol{P}^{\mathrm{H}} \boldsymbol{F}_{\mathrm{d}} \boldsymbol{P}^{*} \boldsymbol{R}^{-1}$.

Moreover, let the inverse frequency response transformation matrix

$\boldsymbol{S}=\boldsymbol{R}^{-1} \boldsymbol{P}^{\mathrm{H}}=\left[S_{i l}, \quad 0 \leqslant i \leqslant N, \quad-M \leqslant l \leqslant M\right]$,

then

$$
\boldsymbol{A}=\boldsymbol{S} \boldsymbol{F}_{\mathrm{d}} \boldsymbol{S}^{\mathrm{H}} .
$$

Now, the remain problem to be solved is to find the closed forms for the elements of the matrix $S$.

\subsection{The elements of the matrix $\boldsymbol{R}$}

By definition,

$$
\boldsymbol{R}-\boldsymbol{P}^{\mathrm{H}} \boldsymbol{P}=\left[R_{i l}, \quad 0 \leqslant i, l \leqslant N\right],
$$

where

$R_{i l}=\sum_{k=-M}^{M} \mathrm{e}^{\mathrm{j} k i \pi / M} \mathrm{e}^{-\mathrm{j} k l \pi / M}$

Depending on the position of the $R_{i l}$ in the matrix, the analysis to obtain simplified expressions is divided into three cases. (only $M>N$ discussed)

Case i. $0 \leqslant i=l \leqslant N$ :

$R_{i l}=\sum_{k=-M}^{M} 1=2 M+1$.
Case ii. $i \neq l$ and $i+l$ even:

$$
\begin{aligned}
R_{i l}=\sum_{k=-M}^{M}[ & \cos \left(\frac{k i \pi}{M}\right) \cos \left(\frac{k l \pi}{M}\right) \\
& +\sin \left(\frac{k i \pi}{M}\right) \sin \left(\frac{k l \pi}{M}\right) \\
& -\mathrm{j} \cos \left(\frac{k i \pi}{M}\right) \sin \left(\frac{k l \pi}{M}\right) \\
& \left.+\mathrm{j} \sin \left(\frac{k i \pi}{M}\right) \cos \left(\frac{k l \pi}{M}\right)\right] .
\end{aligned}
$$

By the analysis in [1], we can obtain

$$
R_{i l}=1 \text {. }
$$

Case iii. $i \neq l$ and $i+l$ odd:

Following (19) and the analysis in [1], we get

$R_{i l}=-1$.

Therefore, we have

$$
\boldsymbol{R}=\left[\begin{array}{ccccc}
2 M+1 & -1 & 1 & -1 & \cdots \\
-1 & 2 M+1 & -1 & 1 & \cdots \\
1 & -1 & 2 M+1 & -1 & \cdots \\
-1 & 1 & -1 & 2 M+1 & \cdots \\
\vdots & \vdots & \vdots & \vdots & \ddots
\end{array}\right]
$$

\subsection{Inversion of the matrix $\boldsymbol{R}$}

Clearly, $\boldsymbol{R}$ can be decomposed into

$$
\begin{aligned}
\boldsymbol{R} & =\left[\begin{array}{ccccc}
2 M & 0 & 0 & \cdots & 0 \\
0 & 2 M & 0 & \cdots & 0 \\
0 & 0 & 2 M & \cdots & 0 \\
\vdots & \vdots & \vdots & \ddots & \vdots \\
0 & 0 & 0 & \cdots & 2 M
\end{array}\right] \\
& +\left[\begin{array}{r}
1 \\
-1 \\
1 \\
-1 \\
\vdots
\end{array}\right]\left[\begin{array}{lllll}
1 & -1 & 1 & -1 & \cdots
\end{array}\right] \\
& =C+\boldsymbol{E E}^{\mathrm{\top}} .
\end{aligned}
$$


Table 1

Elements of the inverse frequency response transformation matrix $S=\left[S_{i l}, 0 \leqslant i \leqslant N,-M \leqslant l \leqslant M\right]$

\begin{tabular}{lll}
\hline$i$ & $l$ & $S_{i l}$ \\
\hline 0 & 0 & $f_{1}$ \\
0 & $-M$ or $M$ & $\frac{1}{2 M+N+1}$ \\
0 & $-M<l<0$ or $0<l<M$ & $\frac{2 M+N-f_{2}(l)-\mathrm{j} f_{3}(l)}{2 M(2 M+N+1)}$ \\
Even & 0 & $\frac{f_{1}}{2 M+N+1}$ \\
Even & $-M$ or $M$ & $\frac{(2 M+N+1) \cos (i l \pi / M)-1-f_{2}(l)+\mathrm{j}\left[(2 M+N+1) \sin (i l \pi / M)-f_{3}(l)\right]}{2 M(2 M+N+1)}$ \\
Even & $-M<l<0$ or $0<l<M$ & $\frac{f_{4}}{2 M+N+1}$ \\
Odd & 0 & $\frac{(2 M+N+1) \cos (i l \pi / M)+1+f_{2}(l)+\mathrm{j}\left[(2 M+N+1) \sin (i l \pi / M)+f_{3}(l)\right]}{2 M(2 M+N+1)}$ \\
Odd & $-M$ or $M \quad$ & $\frac{(2 M<l<0 \text { or } 0<l<M}{2 M}$ \\
Odd & $-M<l$ &
\end{tabular}

Table 2

Expressions for the functions used in Table 1

\begin{tabular}{lll}
\hline Function & Even $N$ & Odd $N$ \\
\hline$f_{1}$ & $\frac{2 M+N}{2 M(2 M+N+1)}$ & $\frac{1}{2 M}$ \\
$f_{2}(l)$ & $\frac{\cos ((N+1) l \pi / M)+\cos (N l \pi / M)-\cos (l \pi / M)-1}{2+2 \cos (l \pi / M)}$ & $\frac{-\cos ((N+1) l \pi / M)-\cos (N l \pi / M)-\cos (l \pi / M)-1}{2+2 \cos (l \pi / M)}$ \\
$f_{3}(l)$ & $\frac{\sin ((N+1) l \pi / M)+\sin (N l \pi / M)-\sin (l \pi / M)}{2+2 \cos (l \pi / M)}$ & $\frac{-\sin ((N+1) l \pi / M)-\sin (N l \pi / M)-\sin (l \pi / M)}{2+2 \cos (l \pi / M)}$ \\
$f_{4}$ & $\frac{2 M+N+2}{2 M(2 M+N+1)}$ & $\frac{1}{2 M}$ \\
\hline
\end{tabular}

By Eq. (23) in [4], the inversion of the matrix $\boldsymbol{R}$ can be expanded as

$$
r_{i l}= \begin{cases}\frac{2 M+N}{2 M(2 M+N+1)}, & i=l \\ \frac{-1}{2 M(2 M+N+1)}, & i \neq l \text { and } i+l \text { even } \\ \frac{1}{2 M(2 M+N+1)}, & i \neq l \text { and } i+l \text { odd }\end{cases}
$$

$$
\begin{aligned}
\boldsymbol{R}^{-1} & =\boldsymbol{C}^{-1}-\boldsymbol{C}^{-1} \boldsymbol{E}\left(1+\boldsymbol{E}^{\mathrm{T}} \boldsymbol{C}^{-1} \boldsymbol{E}\right)^{-1} \boldsymbol{E}^{\mathrm{T}} \boldsymbol{C}^{-1} \\
& =\left[r_{i l}, \quad 0 \leqslant i, l \leqslant N\right] .
\end{aligned}
$$

After some simple manipulation,

\subsection{Elements of $\boldsymbol{S}$ and the coefficients matrix $\boldsymbol{A}$}

Once $\boldsymbol{R}^{-1}$ has been evaluated, the elements of the matrix $S$ can be simplified after some simple 
manipulation. The expressions for the elements of $\boldsymbol{S}$ are given in Table 1, where the expressions for the functions used in this table can be obtained from Table 2.

When the elements in the matrix $S$ have been evaluated by Tables 1 and 2, the elements of $A$ can be obtained by (15)

$a_{l l}=\sum_{k_{1}=-M}^{M} \sum_{k_{2}=-M}^{M} S_{l k_{1}} F_{\mathrm{d}}\left(\frac{k_{1} \pi}{M}, \frac{k_{2} \pi}{M}\right) S_{l k_{2}}$.

Notice that for designing any $N_{1} \times N_{2}$ complex FIR filters, $N$ in Tables 1 and 2 can be only replaced by appropriate $N_{1}$ and $N_{2}$ for evaluating the elements of $S$.

\section{Design examples}

One of the advantages of the linear-phase FIR filters is that they have a constant group delay for all frequencies. Minimum phase FIR filters can be designed to cause less delay, but they can introduce delay distortion because the delay is not a constant for all frequencies. In this section, two examples are presented to demonstrate the designed filters that have less delay than linear-phase filters and have approximately constant group delay in the filter passband. Moreover, in Example 3 an all-pass filter is designed, which is generally used as an equalizer for communications.

Example 1. A $20 \times 20$ circular symmetric lowpass filter with constant group delays $\tau_{1}\left(\omega_{1}, \omega_{2}\right)=$ $\tau_{2}\left(\omega_{1}, \omega_{2}\right)=8$ in the passband is designed in this example. The desired frequency response is given by

$F_{\mathrm{d}}\left(\omega_{1}, \omega_{2}\right)= \begin{cases}\mathrm{e}^{-\mathrm{j} 8 \omega_{1}} \mathrm{e}^{-\mathrm{j} 8 \omega_{2}} & \text { for } \sqrt{\omega_{1}^{2}+\omega_{2}^{2}} \leqslant 0.4 \pi, \\ 0 & \text { for } \sqrt{\omega_{1}^{2}+\omega_{2}^{2}} \geqslant 0.52 \pi\end{cases}$

whose desired amplitude response is illustrated in Fig. 1(a). Notice that the magnitude in transition band is supposed to vary linearly between 1 and 0 . When $M=50$ is used, the amplitude response is shown in Figs. 1(b) and Figs. 1(c) and (d) present the delay responses by $\omega_{1}$ - and $\omega_{2}$-axis, respectively, where we have set the delay response in stopband and transition band to zero for simplification. It is noted that due to the quadrantal symmetry for magnitude and delay responses, the filter coefficients are real. This example took $34 \mathrm{~s}$ CPU time on VAX 8700 , which is faster than $1837 \mathrm{~s}$ spent in eigenfilter approach [6].

Example 2. A $22 \times 22$ complex filter with specifications given in Fig. 2(a) is designed such that it has different constant delay responses in each passband. Also, we used $M=50$, and the amplitude response with circular/square shape flat and conic passbands is shown in Fig. 2(b), in which the regions with horizontal cross lines are the stopbands with desired response 0 , and those with northeast diagonal lines are the passbands with desired response 1 , and those with double cross diagonal lines are conic passbands with maximum response 1 , and the others are the transition bands in which the magnitude varies linearly between 1 and 0 . The delay responses by $\omega_{1}$ - and $\omega_{2}$-axis are shown in Figs. 2(c) and (d), respectively, where we set the delay response in the stopbands and transition bands to zero too. This work took $42 \mathrm{~s}$ on VAX 8700 , and $2146 \mathrm{~s}$ were spent if eigenfilter approach [6] was applied.

Example 3. A $19 \times 19$ 2-D all-pass filter with desired delay responses

$\tau_{1}\left(\omega_{1}, \omega_{2}\right)=-2 \cos \left(\omega_{1}\right) \cos \left(\omega_{2}\right)+9$

and

$\tau_{2}\left(\omega_{1}, \omega_{2}\right)=2 \sin \left(\omega_{1}\right) \sin \left(\omega_{2}\right)+9$

is designed. When $M=50$ is used, Fig. 3(a) shows the amplitude response while delay responses are shown in Figs. 3(b) and (c). The peak amplitude error $\delta=5 \times$ $10^{-5}$, and it took $29 \mathrm{~s}$ on VAX 8700 . Also, the work took long design timc, about $1594 \mathrm{~s}$, for eigenfilter approach [6].

From the above examples, the design time is one or several orders of magnitude small than what it is when other techniques are used. Moreover, the design time is less sensitive to an increase in the order of the filters [1]. 

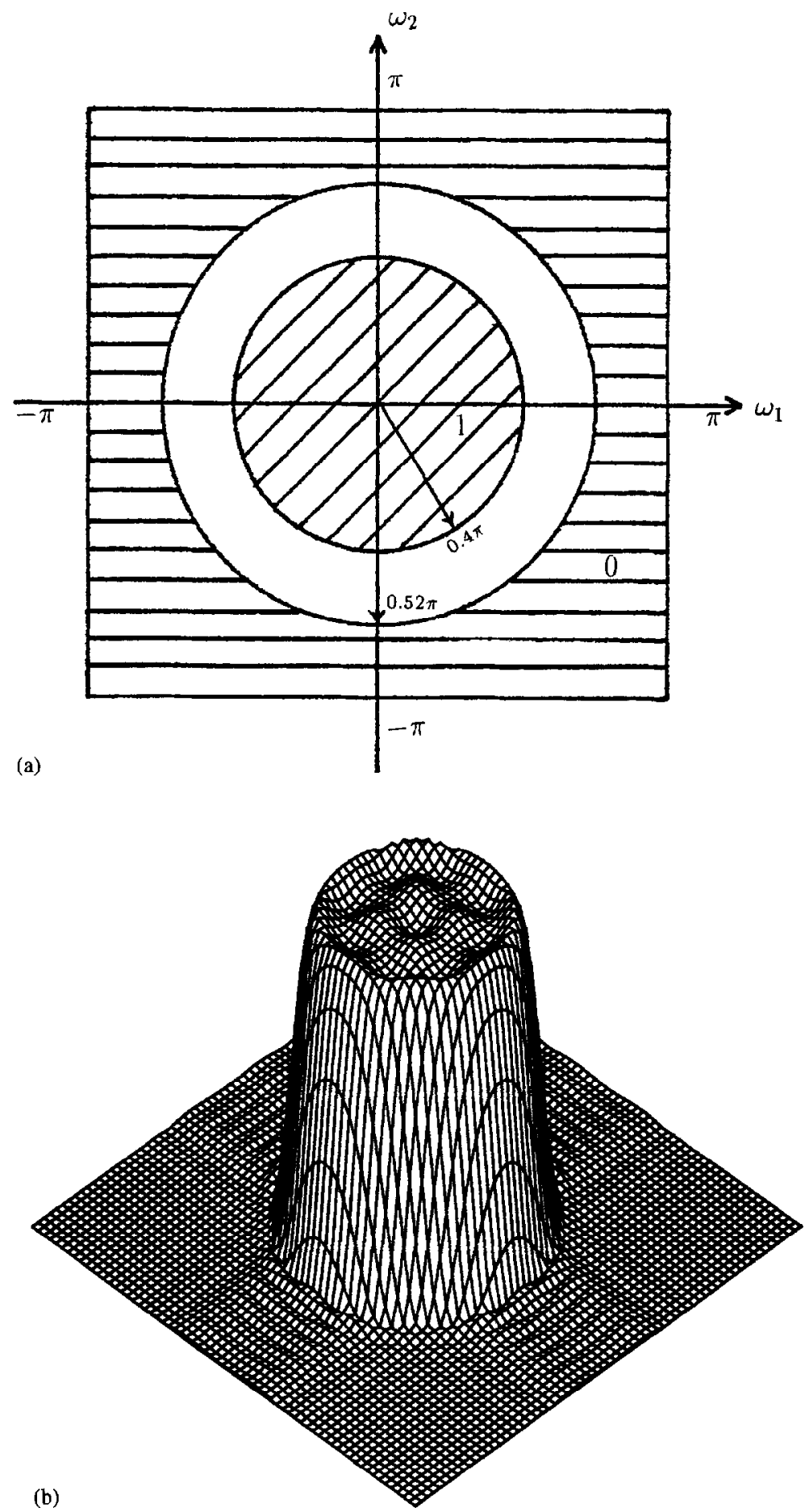

Fig. 1. Example 1. Design of a circular low-pass filter. (a) Specifications, (b) amplitude response. 

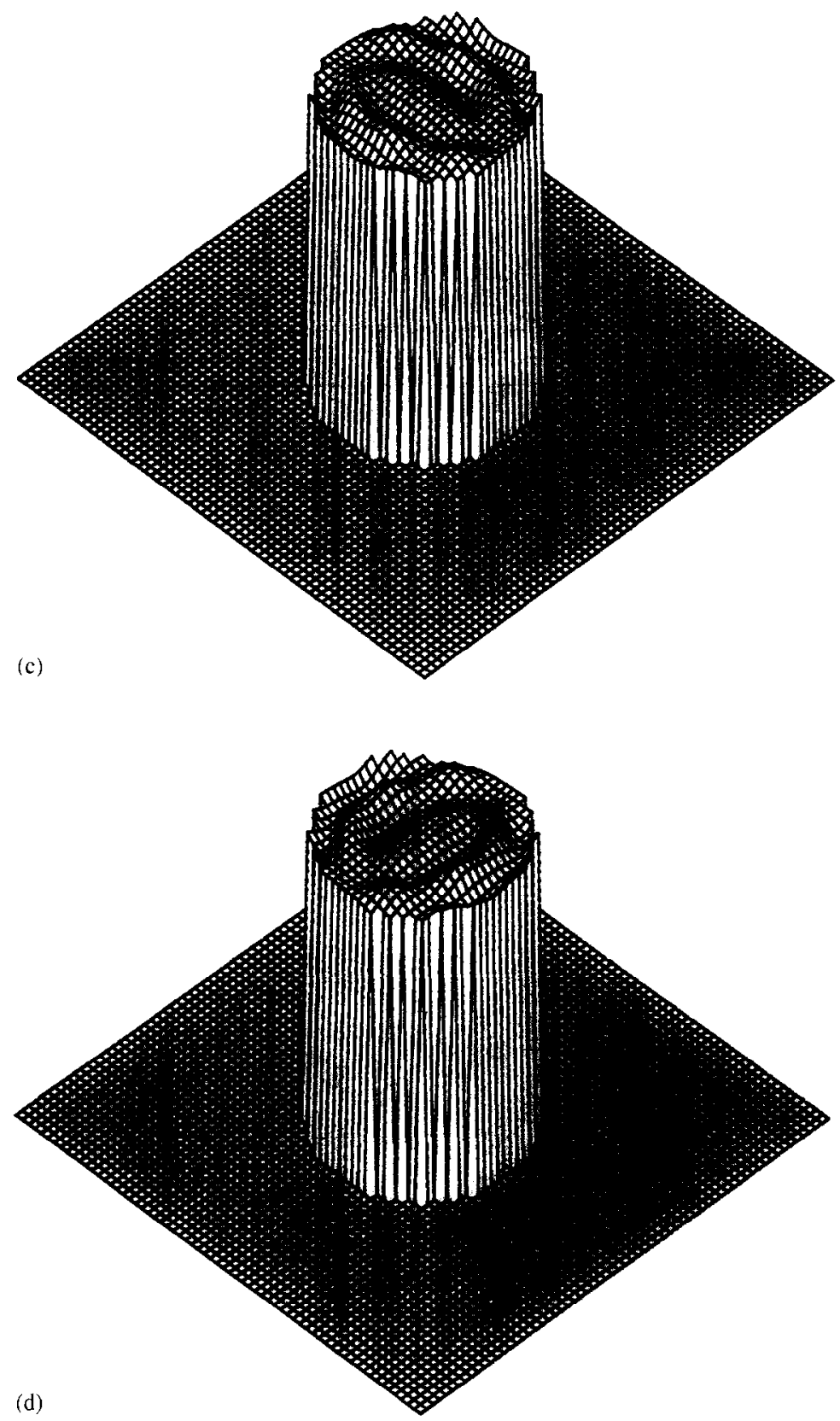

Fig. J. (c) Group delay by $\omega_{1}$-axis, (d) group delay by $\omega_{2}$-axis. 

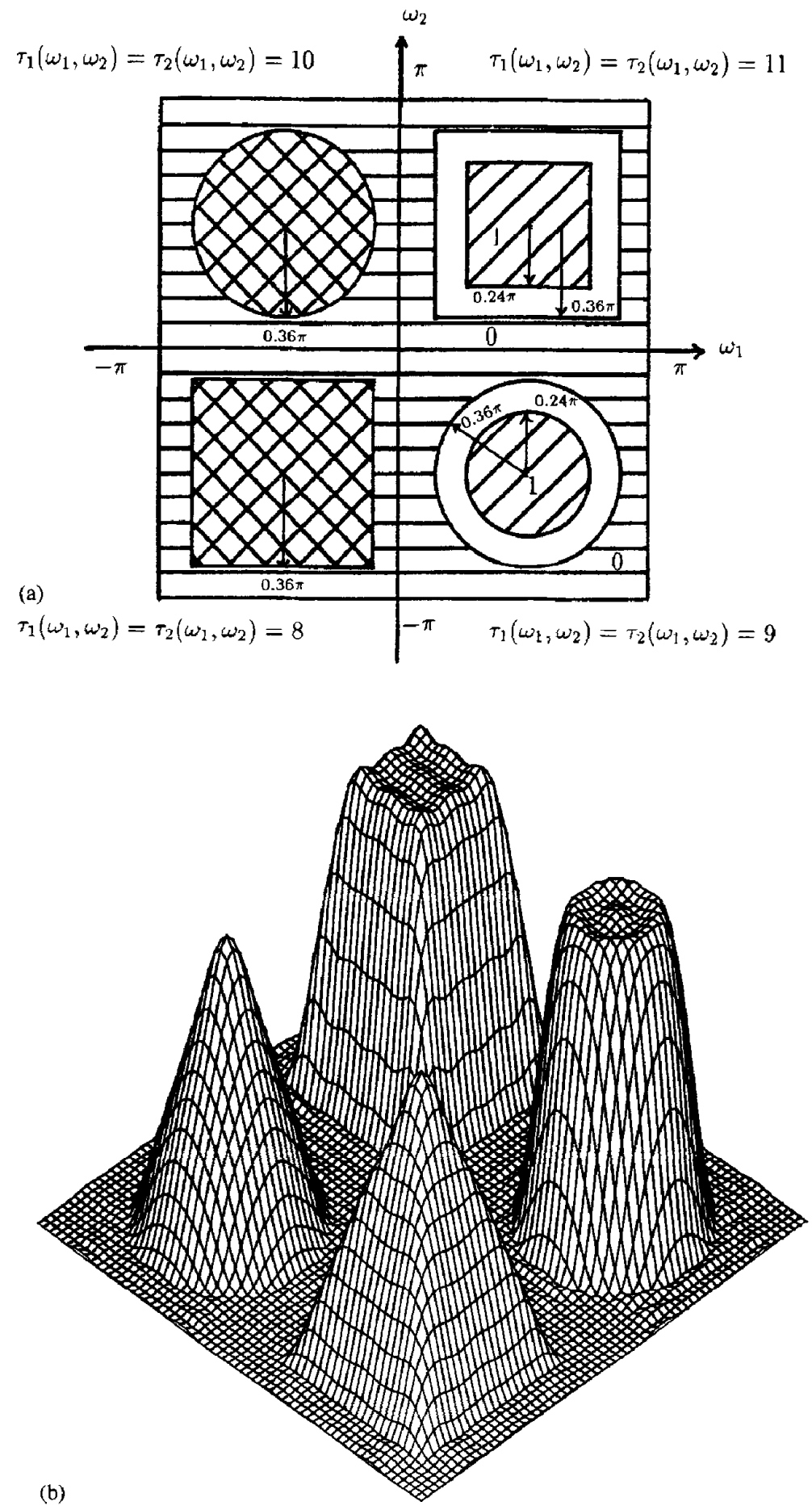

Fig. 2. Example 2. Design of a complex 2-D filter with circular/square shape flat and conic passbands. (a) Specifications, (b) amplitude response. 

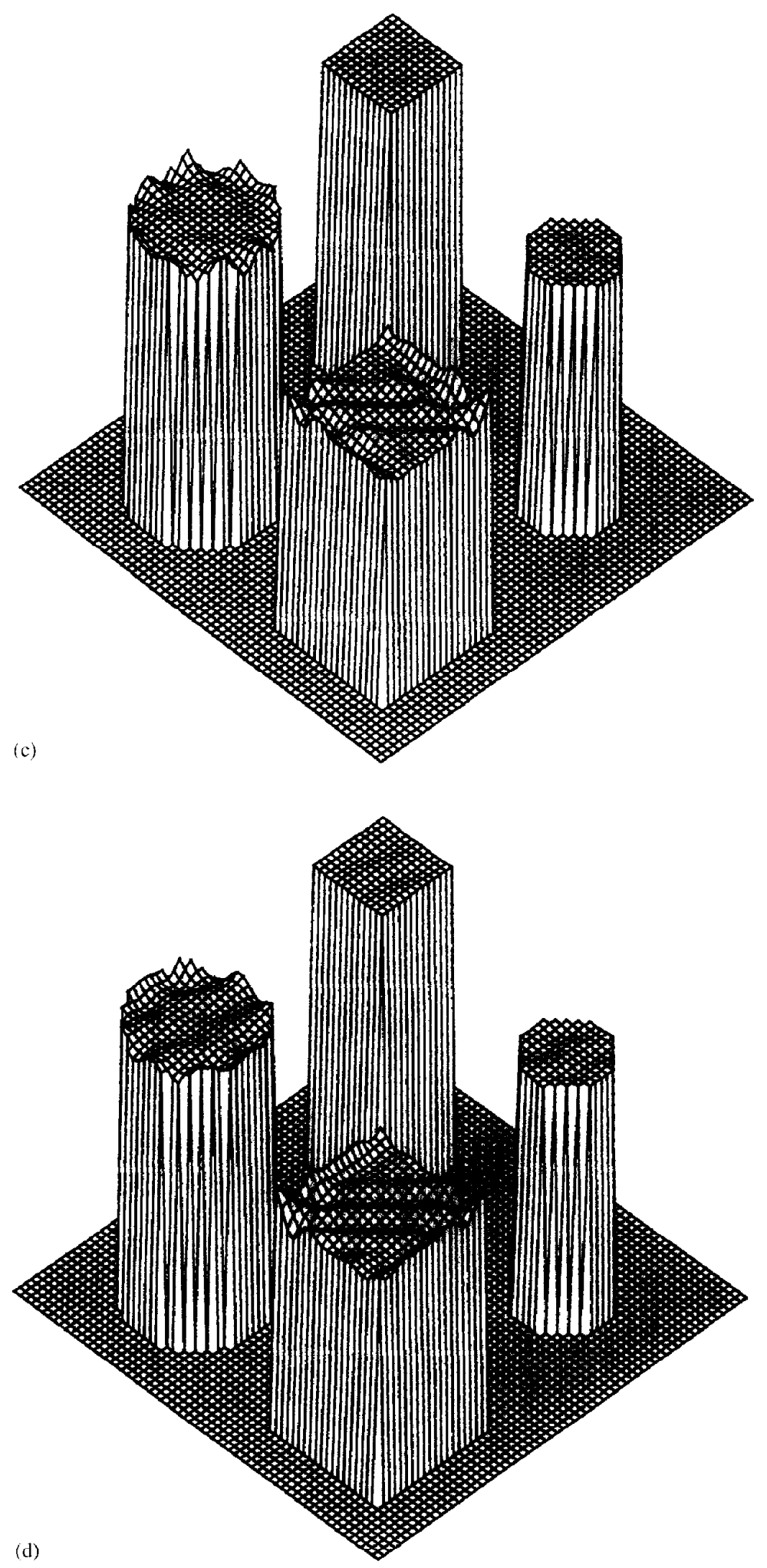

Fig. 2. (c) Group delay by $\omega_{1}$-axis, (d) group delay by $\omega_{2}$-axis. 

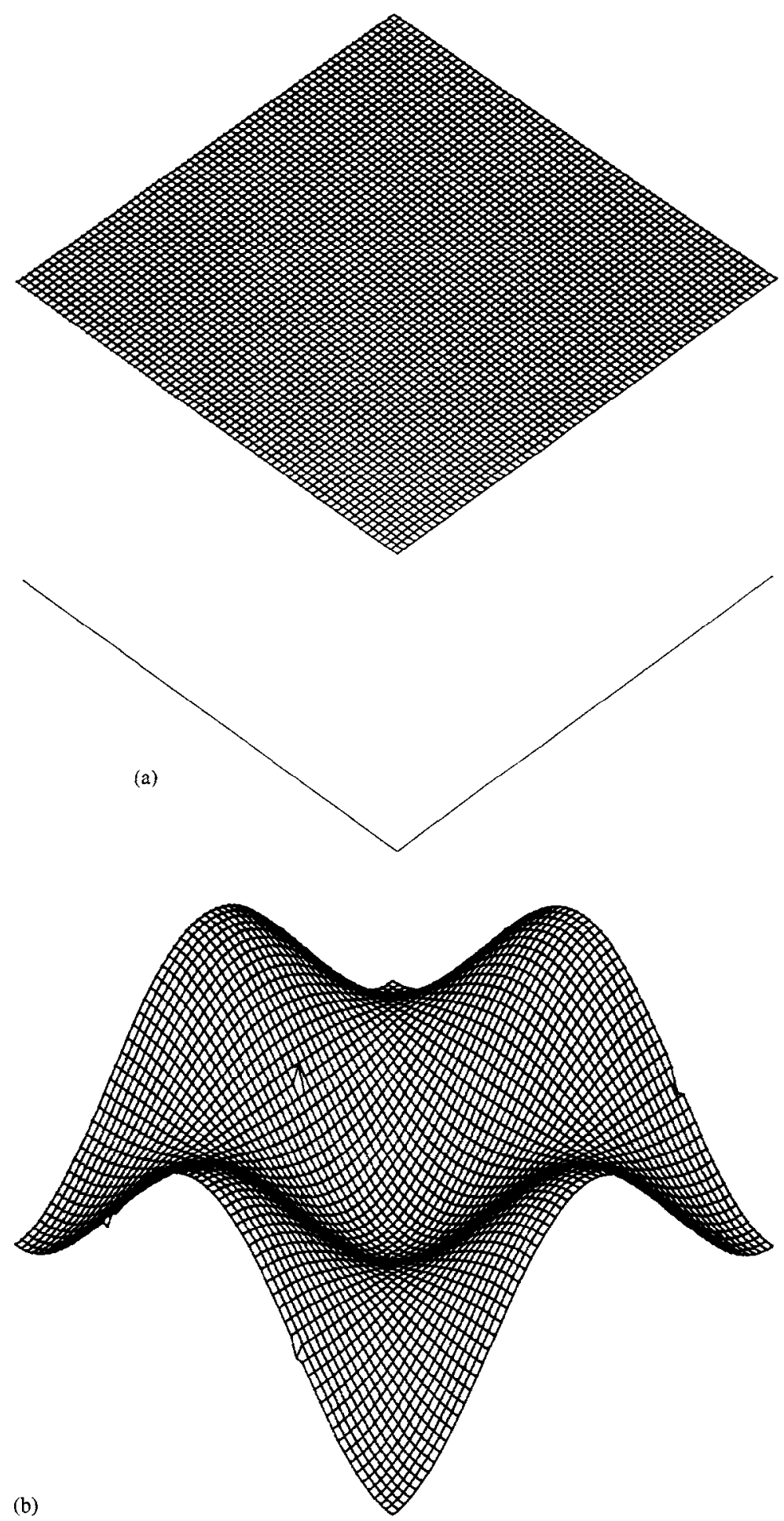

Fig. 3. Example 3. Design of a 2-D all-pass filter. (a) Amplitude response, (b) group delay by $\omega_{1}$-axis. 


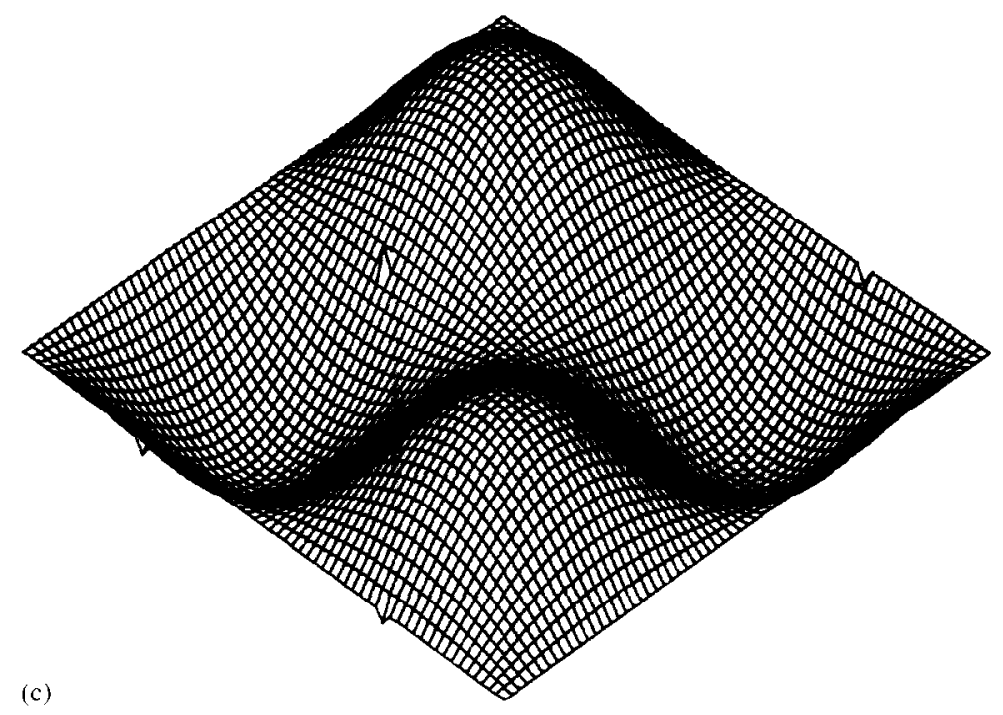

Fig. 3. (c) Group delay by $\omega_{2}$-axis.

\section{Conclusions}

A fast algorithm for designing 2-D FIR digital filters with specified magnitude and group delay characteristics has been presented in this paper. The present method allows the determination of the filter's coefficients for filter design directly from its frequency response specification without employing the usual time-consuming methods of optimization, matrix inversion, or iterative procedures. Several examples including multiband filters and all-pass filters have been designed satisfactorily to demonstrate the capability and efficiency of the method.

\section{References}

[1] M.O. Ahmad and J.D. Wang, "An analytical least square solution to the design problem of two-dimensional FIR filters with quadrantally symmetric or antisymmetric frequency response", IEEE Trans. Circuit Systems, Vol. 36, July 1989, pp. $968-979$.
[2] A.S. Alkhairy, K.G. Christian and J.S. Lim, "Design and characterization of optimal FIR filters with arbitrary phase", IEEE Trans. Signal Process., Vol, 41, February 1993, pp. $559-572$.

[3] X. Chen and T.W. Parks, "Design of FIR filters in the complex domain", IEEE Trans. Acoust. Speech Signal Process., Vol. 35, February 1987, pp. 144-153.

[4] S.L. Marple, Jr., Digital Spectral Analysis with Applications, Prentice-Hall, Englewood Cliffs, NJ, 1987, Chapter 3.

[5] T.Q. Nguyen, "The design of arbitrary FIR digital filters using the eigenfilter method", IEEE Trans. Signal Process., Vol 41, March 1993, pp. 1128-1139.

[6] S.C. Pei and J.J. Shyu, "Complex eigenfilter design of arbitrary complex coefficient FIR digital filters", IEEE Trans. Circuits Systems 1I, Vol. 40, January 1993, pp. 32-40.

[7] K. Preuss, "On the design of FIR filters by complex Chebyshev approximation", IEEE Trans. Acoust. Speech Signal Process., Vol. 37, May 1989, pp. 702-712.

[8] K. Steiglitz, "Design of FIR digital phase networks", IEEE Trans. Acoust Speech Signal Process, Vol. 29, April 1981, pp. $171-176$. 